\title{
脉冲电场下 $\mathrm{ZnO}$ 压敏陶瓷动态击穿过程研究
}

\author{
祝志祥 ${ }^{1}$, 张 强 ${ }^{1}$, 朱思宇 ${ }^{2}$, 卢成嘉 ${ }^{3}$, 刘 乙 $^{3}$, 杨 佳 $^{3}$, \\ 吴超峰 ${ }^{4}$, 曹林洪 ${ }^{2}$, 王 辑 ${ }^{4}$, 高志鹏 ${ }^{3}$, 朱承治 ${ }^{5}$
}

(1. 全球能源互联网研究院有限公司电工新材料研究所, 先进输电技术国家重点实验室, 北京 $102211 ; 2$. 西南科 技大学 材料科学与工程学院, 绵阳 $621000 ; 3$. 中国工程物理研究院 流体物理研究所, 冲击波物理与爆轰物理重 点实验室, 绵阳 621900; 4. 清华大学 材料科学与工程学院, 北京 100084; 5. 国网浙江省电力有限公司, 杭州 310007)

摘 要: 陶瓷电击穿问题涉及热、光、电多场耦合效应，一直是非平衡物理学研究的重点和热点。本工作在不同烧 结温度下制备了晶粒尺寸大小不同的氧化锌陶瓷, 采用脉冲高压发生装置对陶瓷进行击穿实验, 通过对陶瓷击穿 过程的分析和对比, 研究了 $\mathrm{ZnO}$ 陶瓷体击穿的时间步骤。结果显示, 不同晶粒大小的陶瓷击穿过程均在 $7 \mu \mathrm{s}$ 之内, 典型的压降曲线分为三个阶段。第一个阶段对应于材料中的气孔击穿和击穿通道初步形成; 第二阶段对应于晶界击 穿; 第三个阶段是导电通道的完全形成。研究数据显示, 晶粒击穿过程的持续时间最长, 晶界次之, 气孔的击穿时 间最短。不同烧结温度下, 样品晶界和晶粒的击穿时间以及气孔的击穿速度均存在差异。

关 键 词: $\mathrm{ZnO}$ 陶瓷; 多晶材料; 击穿过程; 脉冲电压

中图分类号: TN304 文献标识码: A

\section{Dynamic Breakdown of ZnO Varistor Ceramics under Pulsed Electric Field}

\author{
ZHU Zhi-Xiang ${ }^{1}$, ZHANG Qiang ${ }^{1}$, ZHU Si-Yu ${ }^{2}$, LU Cheng-Jia ${ }^{3}$, LIU Yi $^{3}$, YANG Jia $^{3}$, \\ WU Chao-Feng ${ }^{4}$, CAO Lin-Hong ${ }^{2}$, WANG Ke ${ }^{4}$, GAO Zhi-Peng ${ }^{3}$, ZHU Cheng-Zhi ${ }^{5}$
}

(1. State Key Laboratory of Advanced Power Transmission Technology, Institute of New Electrical Materials, Global Energy Interconnection Research Institute, Beijing 102211, China; 2. School of Materials Science and Engineering, Southwest University of Science and Technology, Mianyang 621000, China; 3. Laboratory of Shock Wave and Detonation Physics, Institute of Fluid Physics, China Academy of Engineering Physics, Mianyang 621900, China; 4. School of Materials Science and Engineering, Tsinghua University, Beijing 100084, China; 5. State Grid Zhejiang Electric Power Co., Ltd., Hangzhou 310007, China)

\begin{abstract}
Electrical breakdown process of ceramics is a complex process related to electrical, thermal, and light effects. It has attracted lots of attentions. But the mechanism of dielectric breakdown is still under debate, especially for the solid ceramics. In this study, the ceramics with different grain size were sintered at different temperatures. A square pulsed voltage of $27 \mathrm{kV}$ with the pulse width of $7 \mu$ s was generated to breakdown the $\mathrm{ZnO}$ ceramics with a thickness of $2 \mathrm{~mm}$. Electric breakdown process of these ceramics were investigated using a homemade high-timeresolution electric detection system with the resolution $<1 \mathrm{~ns}$. Compared the electric behaviors of $\mathrm{ZnO}$ ceramics with different grain sizes during the breakdown process, it is clear that the whole electric breakdown process could be divided into three main steps, all of which can completed within $7 \mu$ s. The first breakdown step is the pores breakdown due to the electric field concentration, and the second step is the grain boundaries breakdown, and then the
\end{abstract}

收稿日期：2018-09-14; 收到修改稿日期：2018-11-12

基金项目: 国家电网公司科技项目(52110417000S) National Power Grid Corp Science and Technology Project (52110417000S) 作者简介: 祝志祥(1981-), 男, 博士.E-mail: zhuzhixiang003@163.com

通讯作者: 王 轲, 副教授. E-mail: wang-ke@tsinghua.edu.cn; 高志鹏, 副研究员. E-mail: z.p.gao@foxmail.com 
bulk grain is broken-down leading to form the whole electrical breakdown channel. The data shows that the breakdown process of the grains has the longest duration, the grain boundary is the second, and the pores breakdown is the shortest. For the ceramics with different grain sizes, the breakdown durations of the grain boundaries are different. The duration and electrical breakdown speed of the three step processes are directly affected by the resistance uniformity and carrier concentration of the materials.

Key words: $\mathrm{ZnO}$ ceramics; polycrystalline materials; breakdown process; pulse voltage

1967 年日本松下公司首先开发出 $\mathrm{ZnO}-\mathrm{Bi}_{2} \mathrm{O}_{3}$ 系 压敏陶瓷 ${ }^{[1]}$, 由于其优异的非线性系数、极快的响应 速度、较高的通流能力以及低廉的工艺成本, 被广 泛应用于电过载保护领域, 如高压输电系统中的电 涌保护器以及防雷系统中的避雷器等。在这些高压 环境的应用中，压敏电阻与被保护装置并联，当用 电器两端电压骤升时, 压敏电阻的阻值会迅速减小, 使电路产生瞬间短路, 起到保护作用。压敏电阻在 工程应用中需时常承受较高的电压, 导致 $\mathrm{ZnO}$ 压敏 陶瓷的击穿现象非常普遍, 为了避免压敏电阻被击 穿所引起的输电线路故障以及雷击事故, 对压敏电 阻击穿现象的研究在工程应用中具有重大意义 ${ }^{[2-3]}$ 。

陶瓷体中存在气孔、晶粒、晶界等结构, 其电 击穿过程涉及到热效应, 静力学效应, 动力学效 应 $^{[4]}$ 等, 目前还没有一个标准的模型来解释陶瓷的 击穿问题。大量研究表明, 在电场作用下, 材料被击 穿时会在表面或内部形成一条或多条击穿通道(又 称导电通道), 因此光学方法成为观测击穿通道的 动态变化特征的主要方法, 并借此来研究透明电介 质材料的电击穿过程。然而许多陶瓷材料不具有透 光特性, 很难用光学方法直接观察其击穿通道变 化。在针对不透光陶瓷体击穿过程的研究中, 陈艺 灵等 ${ }^{[5]}$ 采用高速摄像机观察了高压陶瓷电容材料的 击穿过程, 但该方法只能记录发生在电极表面以及 边沿的放电、燃烧等现象, 无法针对陶瓷内部的击 穿过程进行观测和分析。刘艺等 ${ }^{[6]}$ 搭建了微秒脉冲 高压发生装置, 通过测量 PZT95/5 陶瓷在脉冲电场 下等效电阻的变化, 发现该陶瓷的体击穿可分为两 个阶段, 分别为孔洞、微裂纹击穿和晶粒击穿, 且体 击穿导电通道的形成速率大于沿面击穿阶段。目前, 对于 $\mathrm{ZnO}$ 压敏陶瓷击穿现象的研究主要集中于通 过陶瓷粉体的改性, 从而提升陶瓷体的密度和均匀 性 $^{[7-8]}$ 或者降低晶粒电阻 ${ }^{[9-11]}$, 优化其在脉冲电流冲 击下的稳定性和能量耐受量, 而对于击穿过程本身 的探索还有待完善。本研究采用微秒脉冲高压发生 装置对不同温度下烧结的 $\mathrm{ZnO}$ 压敏陶瓷样品进行 脉冲电压加载, 分析对比了二者在击穿过程中的压
降曲线的变化特征的异同，得到了 $\mathrm{ZnO}$ 压敏陶瓷各 部分结构被击穿的先后顺序, 并对影响各击穿过程 中时间和速度的因素做了分析, 为未来针对 $\mathrm{ZnO}$ 压 敏陶瓷材料物性的进一步研究提供了思路。

\section{1 实验方法}

实验所用 $\mathrm{ZnO}$ 压敏陶瓷采用分析纯(99\%)的原 料进行配料, 配方比例为 $94.8 \mathrm{~mol} \% \mathrm{ZnO}, 1.7 \mathrm{~mol} \%$ $\mathrm{Sb}_{2} \mathrm{O}_{3}, 0.6 \mathrm{~mol} \% \mathrm{Bi}_{2} \mathrm{O}_{3}, 0.8 \mathrm{~mol} \% \mathrm{Co}_{3} \mathrm{O}_{4}, 1.2 \mathrm{~mol} \%$ $\mathrm{NiO}, 0.62 \mathrm{~mol} \% \mathrm{MnO}, 0.28 \mathrm{~mol}_{\%} \% \mathrm{SiO}_{2}$, 湿法球磨混 合均匀并干燥后得到 $\mathrm{ZnO}$ 压敏陶瓷粉体。添加质量 分数为 $10 \%$ 的聚乙烯醇(PVA)溶液。经手工造粒后, 压制成圆状生坏。生坏经排塑后, 以 $3{ }^{\circ} \mathrm{C} / \mathrm{min}$ 的升 温速率分别在 1000 和 $1150{ }^{\circ} \mathrm{C}$ 烧结 $2 \mathrm{~h}$, 对烧结后的 样品进行打磨、超声、刷银, 在 $590{ }^{\circ} \mathrm{C}$ 保温 $20 \mathrm{~min}$ 烧银, 最后获得直径为 $15 \mathrm{~mm}$, 厚度约 $8 \mathrm{~mm}$ 的试 样。本文中, 为了方便描述, 分别将在 1000 和 $1150{ }^{\circ} \mathrm{C}$ 烧结的 $\mathrm{ZnO}$ 陶瓷样品简称为 $1000{ }^{\circ} \mathrm{C}$ 样品和 $1150{ }^{\circ} \mathrm{C}$ 样品。

$\mathrm{ZnO}$ 压敏陶瓷的断面形貌采用扫描电子显微镜 (TM3000, 日本日立)进行观察。材料的相结构组成 采用 X 射线衍射仪(Dmax-RB, 日本理学)进行表征。 研究中采用了自主搭建的高时间分辨电子测试系统 $(I F P, C A E P)^{[6]}$ 对不同烧结温度的 $\mathrm{ZnO}$ 压敏陶瓷进行 脉冲击穿测试。测试平台的时间分辨率为小于 $1 \mathrm{~ns}$ 。

\section{2 结果与讨论}

图 1 为 1000 和 $1150{ }^{\circ} \mathrm{C}$ 样品的 $\mathrm{X}$ 射线衍射分析 (XRD)图谱。从衍射峰上可以发现, 两个样品的主要 晶相组成均为 $\mathrm{ZnO}$ 相, $\mathrm{Zn}_{7} \mathrm{Sb}_{2} \mathrm{O}_{12}$ 尖晶石相和 $\mathrm{Bi}_{2} \mathrm{O}_{3}$ 相。与其它类似组分 $\mathrm{ZnO}$ 压敏陶瓷的衍射图谱相

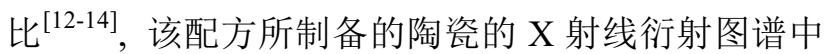
没有出现硅锌矿相, 这可能与配方中 $\mathrm{SiO}_{2}$ 含量较少 有关。另一方面, 配方中掺入的 Co、 $\mathrm{Mn} 、 \mathrm{Ni}$ 主要 存在于晶界 $\mathrm{Zn}_{7} \mathrm{Sb}_{2} \mathrm{O}_{12}$ 尖晶石相和 $\mathrm{Bi}_{2} \mathrm{O}_{3}$ 相中, 其主 要作用是改善材料的电性能, 并没有改变陶瓷体的 
相结构组成, 这与参考文献中结果相一致 ${ }^{[15]}$ 。对比 不同温度下烧结的 $\mathrm{ZnO}$ 压敏陶瓷的衍射峰, 由于配 方相同, 二者的 $\mathrm{ZnO}$ 相的峰强几乎一致, 虽然 $1150{ }^{\circ} \mathrm{C}$ 样品的 $\mathrm{ZnO}$ 晶粒更大，但是二者 $\mathrm{ZnO}$ 相衍 射峰的半高宽并没有体现出明显差异。这主要是由 于通常情况下, XRD 衍射峰半高宽的变化能反应晶 粒尺寸 $<100 \mathrm{~nm}$ 的晶粒大小变化 ${ }^{[16]}$, 而本次研究中 的 $\mathrm{ZnO}$ 压敏陶瓷的晶粒大多是微米级的。另一方面, $1150{ }^{\circ} \mathrm{C}$ 样品的 $\mathrm{Zn}_{7} \mathrm{Sb}_{2} \mathrm{O}_{12}$ 尖晶石相衍射峰的强度有 轻微的增大，原因在于部分 $\mathrm{Zn}_{7} \mathrm{Sb}_{2} \mathrm{O}_{12}$ 尖晶石相是 在温度达到 $900{ }^{\circ} \mathrm{C}$ 以上时由焦绿石相转变而来, 该 反应同时析出了 $\mathrm{Bi}_{2} \mathrm{O}_{3}$, 反应方程式如(1)所示。当烧 结温度为 $1000{ }^{\circ} \mathrm{C}$ 时, 生成尖晶石相的反应不够彻 底, 所以其 $\mathrm{Zn}_{7} \mathrm{Sb}_{2} \mathrm{O}_{12}$ 尖晶石相衍射峰强低于 $1150{ }^{\circ} \mathrm{C}$ 烧结的样品。同时, $1000{ }^{\circ} \mathrm{C}$ 样品的 $\mathrm{Bi}_{2} \mathrm{O}_{3}$ 相 的衍射峰较 $1150{ }^{\circ} \mathrm{C}$ 样品更强, 这是因为析出的 $\mathrm{Bi}_{2} \mathrm{O}_{3}$ 液相随着烧结温度的升高出现了挥发现象, 导致了衍射峰的衰减。

$$
\begin{aligned}
& 2 \mathrm{Zn}_{2} \mathrm{Bi}_{3} \mathrm{Sb}_{3} \mathrm{O}_{14}+17 \mathrm{ZnO} \rightarrow \\
& \quad 3 \mathrm{Zn}_{7} \mathrm{Sb}_{2} \mathrm{O}_{12}+3 \mathrm{Bi}_{2} \mathrm{O}_{3} \text { (Liquid) }
\end{aligned}
$$

图 2 为在 1000 和 $1150{ }^{\circ} \mathrm{C}$ 样品断面的 SEM 照片, 图中的白色方框标识了材料中的气孔, 而椭圆圈标 识晶界相。可以看出, $\mathrm{Zn}_{7} \mathrm{Sb}_{2} \mathrm{O}_{12}$ 尖晶石相和 $\mathrm{Bi}_{2} \mathrm{O}_{3}$ 相均偏析在晶界。其中 $\mathrm{Bi}_{2} \mathrm{O}_{3}$ 相作为晶界的骨架, 固 溶有许多 Co、 $\mathrm{Mn} 、 \mathrm{Ni}$ 元素 ${ }^{[17]}$ ，也是 $\mathrm{ZnO}$ 压敏陶瓷 的非线性 $U-I$ 特性的来源 ${ }^{[18]} 。 \mathrm{Zn}_{7} \mathrm{Sb}_{2} \mathrm{O}_{12}$ 尖晶石相则 起到“钉扎作用”，防止晶粒异常长大，并且该尖晶 石相在生长过程中使掺杂的 Co、Mn、Ni 元素均匀 分布 ${ }^{[19-20]}$ 。从晶粒大小来看, $1150{ }^{\circ} \mathrm{C}$ 样品的晶粒远 大于 $1000{ }^{\circ} \mathrm{C}$ 样品, $1150{ }^{\circ} \mathrm{C}$ 样品的晶粒尺寸为 $7 \sim$ $10 \mu \mathrm{m}$, 而 $1000{ }^{\circ} \mathrm{C}$ 样品的晶粒尺寸仅为 $1 \sim 3 \mu \mathrm{m}$ 。这 种差异主要是由于随着烧结温度的升高, 烧结的推 动力增大, 导致晶粒的长大。另外, $1150{ }^{\circ} \mathrm{C}$ 样品的晶

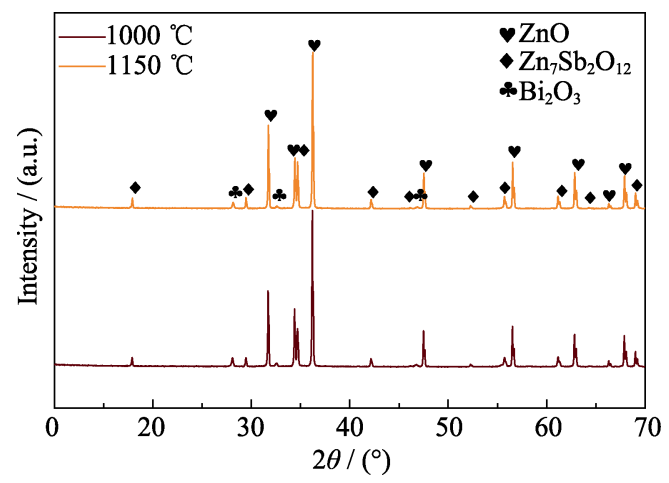

图 1 样品的 XRD 图谱

Fig. 1 XRD patterns of the samples sintered at 1000 and $1150{ }^{\circ} \mathrm{C}$
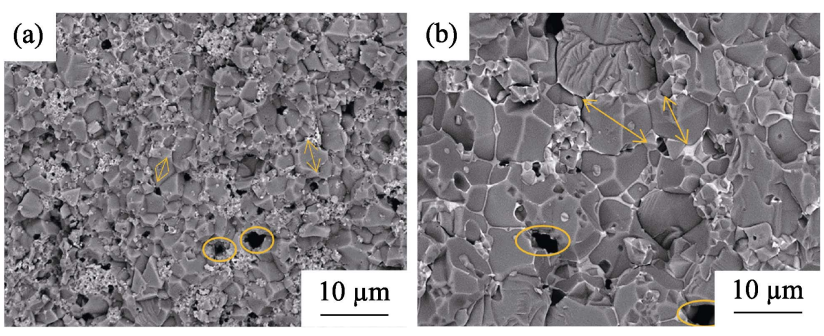

图 2 样品断面的 SEM 照片

Fig. 2 SEM images of the samples sintered at 1000 (a) and $1150{ }^{\circ} \mathrm{C}$ (b)

粒表面出现了更大的气孔, 且其密度 $\left(5.48 \mathrm{~g} / \mathrm{cm}^{3}\right)$ 低 于 $1000{ }^{\circ} \mathrm{C}$ 样品的密度 $\left(5.56 \mathrm{~g} / \mathrm{cm}^{3}\right)$ 。由图 1 中的 XRD 测试结果可知, $\mathrm{Bi}_{2} \mathrm{O}_{3}$ 随着温度的升高析出并挥发造 成大气孔产生及密度下降的原因之一。除此之外, 更高温度下晶界的移动和气孔合并对气孔的长大也 有直接贡献。

1000 和 $1150{ }^{\circ} \mathrm{C}$ 样品的稳压电位梯度大概分别 为 2.4 和 $1.6 \mathrm{kV} / \mathrm{mm}$ 。为了重点研究材料的击穿过 程, 采用最高电压为 $27 \mathrm{kV}$, 长度为 $7 \mu \mathrm{s}$ 的脉冲电压 对样品进行击穿测试, 实验等效电路与加载电压波 形如图 3(a) (b)所示。由于加载电场远高于电位梯 度, 故本研究中可以不考虑电位梯度对击穿行为的 影响。在图 3(a)中, $\mathrm{T}$ 为调压器, $\mathrm{D}$ 为整流二极管, $R_{1}$ 为充电电阻, $C$ 为脉冲电容, $K$ 为触发开关, $R_{2}$ 为限 流电阻, $R_{3}$ 为保护电阻, $R_{\mathrm{S}}$ 为负载(陶瓷样品), 其中 $R_{2}$ 为 $400 \Omega$ 。实验装置采用 25 号变压器油作为绝缘 保护介质。一般而言, 击穿通道形成和发展的过程 可简单地用材料等效电阻的变化过程来表征。陶瓷 样品在发生电击穿前相当于陶瓷电容器, 表现为非 线性介质, 随着击穿过程中导电通道的形成, 样品 的电阻特性占据主导作用, 此时可近似作为线性电 介质分析其等效电阻的变化。根据实验电路模型, 陶瓷样品电阻 $R_{\mathrm{S}}$ 、样品两端电压 $U_{\mathrm{s}}$ 和装置输出电压 $U$ 在击穿过程中的变化规律可用电路方程描述为:

$$
U=U_{\mathrm{s}} \times\left(1+R_{2} / R_{\mathrm{s}}\right)
$$

基于脉冲高压发生装置设计原理，击穿发生时 外部输出电压 $U$ 基本不变, 即 $\mathrm{d} U / \mathrm{d} t=0$, 由式(2)推 导可得 $R_{\mathrm{S}}$ 与 $U_{\mathrm{s}}$ 的关系为:

$$
R_{\mathrm{S}}=\left(U_{\mathrm{s}} \times R_{2}\right) /\left(U-U_{\mathrm{s}}\right)
$$

式(3)中 $R_{2}$ 和 $U$ 为已知, 因此可以由 $U_{\mathrm{s}}$ 推算出样品 电阻 $R_{\mathrm{S}}$ 随时间的变化 ${ }^{[6]}$ 。

图 4(a) (b)所示为不同烧结温度 $\mathrm{ZnO}$ 压敏陶瓷 样品击穿过程中电场的变化波形, 为了方便讨论, 这里把整个电压加载过程和陶瓷的击穿过程分为 1-7 个区域(图 4(a))。区 1 为电压加载的升压区，我 们可以看出, 1000 和 $1150{ }^{\circ} \mathrm{C}$ 样品都没有在升压过程 

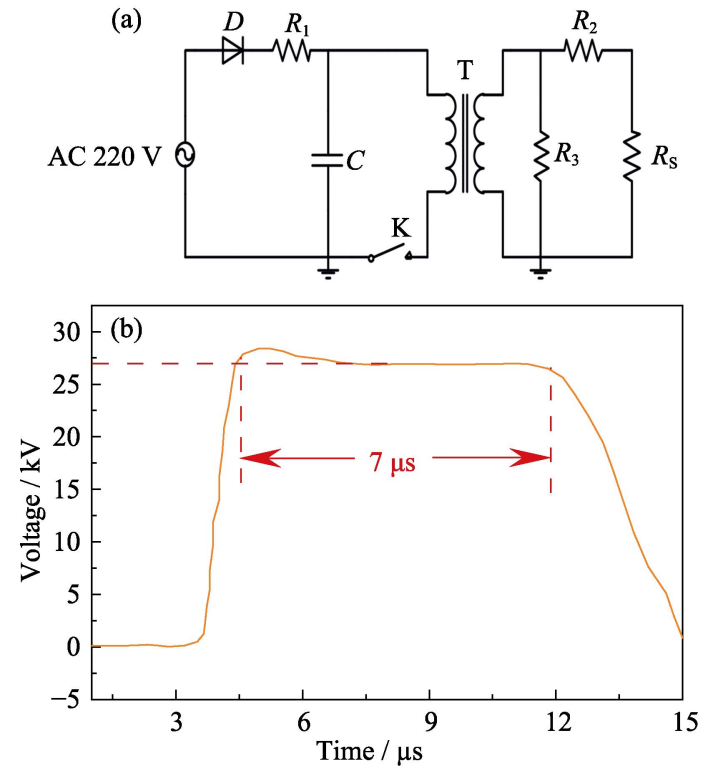

图 3 装置等效电路图(a)和加载电压波形(b)

Fig. 3 Circuit of experimental set-up (a) and applied voltage curve (b)
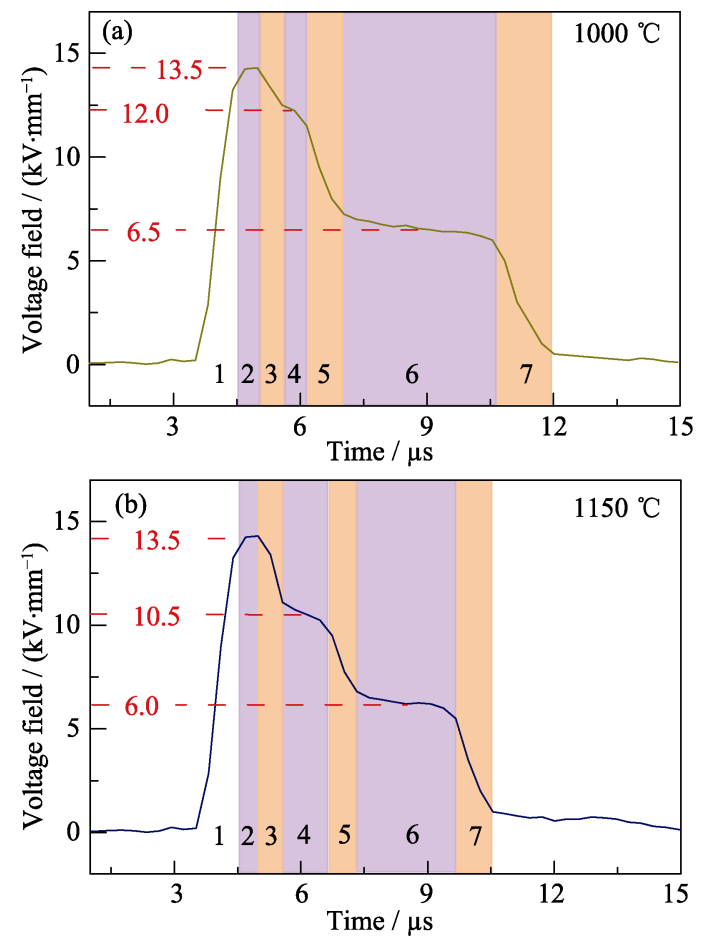

图 4 不同烧结温度陶瓷样品的击穿过程中的电场变化波形, 紫色电压平台区为样品稳定区, 橙色电压下降区为击穿过 程区

Fig. 4 Voltage change during breakdown process

The purple voltage platform area is the sample stable area, and the orange voltage drop area is the breakdown process area

中出现击穿。在加载电场达到 $13.5 \mathrm{kV} / \mathrm{mm}$ 后, 1000 和 $1150{ }^{\circ} \mathrm{C}$ 样品均在 $5 \mu \mathrm{s}$ 左右开始出现击穿, 二者的 击穿电压波形包含三个电压稳定区 $(2,4,6)$ 和三个 电压下降区 $(3,5,7)$ 。 $1000{ }^{\circ} \mathrm{C}$ 样品和 $1150{ }^{\circ} \mathrm{C}$ 样品在
击穿区 3 中的电压梯度降最小, 分别从初始加载 电场强度 $13.5 \mathrm{kV} / \mathrm{mm}$ 下降到了 12 和 $10.5 \mathrm{kV} / \mathrm{mm}$, 压降分别为 1.5 和 $3 \mathrm{kV} / \mathrm{mm}$ 。击穿区 5 中, $1000{ }^{\circ} \mathrm{C}$ 样品的加载电位梯度从 $12 \mathrm{kV} / \mathrm{mm}$ 下降到了 $6.5 \mathrm{kV} / \mathrm{mm}$, 电位梯度降为 $5.5 \mathrm{kV} / \mathrm{mm}$ 。 $1150{ }^{\circ} \mathrm{C}$ 样品 的电场强度从 $10.5 \mathrm{kV} / \mathrm{mm}$ 下降到了 $6 \mathrm{kV} / \mathrm{mm}$, 电位 梯度降为 $4.5 \mathrm{kV} / \mathrm{mm}$; 而在击穿区 7 中, $1000{ }^{\circ} \mathrm{C}$ 样品 和 $1150{ }^{\circ} \mathrm{C}$ 样品的电位梯度降基本相同, 分别为 6.5 和 $6 \mathrm{kV} / \mathrm{mm}$ 。

为了进一步说明材料的击穿机制, 根据公式 (2) (3), 可以得出陶瓷样品电阻在击穿过程中随时 间的变化, 如图 5 所示。根据曲线特征, 与电场变化 相一致, 样品的电阻变化有三个阶段, 如图中区域 I、II、III 所示。这三个阶段分别对应了图 4 中的 3、 5 和 7 阶段。从图 5 中可以直接看出, 区域 I 是击穿 的初始部分, 这部分发生在击穿刚开始的 $200 \mathrm{~ns}$ 左 右。在这个阶段, 两个样品的电阻均从 $10^{11} \Omega$ 下降 到 $10^{4} \Omega$ 左右。从电阻的数值上可以看出, $10^{4} \Omega$ 大于 晶粒内部的电阻值, 对应晶界的电阻值。区域 II 的 电阻值变化对应于第二次电位降低, 对应于晶界的 击穿, 孔洞或微裂纹击穿后施加在晶界上的电场强 度增大, 加上载流子产生的热效应的逐渐累积, 使 得晶界发生击穿。在这一阶段, 陶瓷的电阻值从 $10^{4} \Omega$ 下降到 $10^{2} \sim 10^{3} \Omega$ 。第三阶段, 在热效应的作 用下, 陶瓷内部的通道进一步扩展, 晶粒晶界完全 贯通, 电阻值进一步下降至 $<10 \Omega$, 并且逐渐趋于 稳定。一般而言, 由于材料内部存在气孔或者裂纹, 使电场非均匀分布, 因此通道裂纹内部的电场与外 加电场的分布不同。正是由于气孔边缘的电势分布 梯度大从而导致能量的集中, 在气孔部位往往最先 发生击穿 ${ }^{[21]}$ 。可以认为击穿区 I 代表着 $\mathrm{ZnO}$ 压敏陶 瓷中气孔的击穿。值得明确的是, 由于陶瓷内部结 构复杂, 这里的气孔击穿必然会伴随着一些晶界和 晶粒的击穿效应。因此, 这里的区域 I 更多地代表 了由气孔击穿诱发的通道开始形成。另外, 由于 $1150{ }^{\circ} \mathrm{C}$ 样品中的气孔更大, 占比更多, 所以在击 穿区 3 中 $1150{ }^{\circ} \mathrm{C}$ 样品的压降大于 $1000{ }^{\circ} \mathrm{C}$ 样品 (图 4)。击穿区 II 为晶界的击穿过程, 由于 $1000{ }^{\circ} \mathrm{C}$ 样品的晶粒小于 $1150{ }^{\circ} \mathrm{C}$ 样品的晶粒, 在厚度相同 的情况下, $1000{ }^{\circ} \mathrm{C}$ 样品晶界更多, 使其击穿电压波 形出现更大的压降。击穿区 III 为 $\mathrm{ZnO}$ 晶粒的击穿 区, 相比于晶界浓度对击穿的影响, $\mathrm{ZnO}$ 晶粒的大 小并不会对其在击穿电压波形中的压降造成显著 影响, 这可能是晶界的电阻率远高于晶粒的电阻 率所致 ${ }^{[22-23]}$ 。 

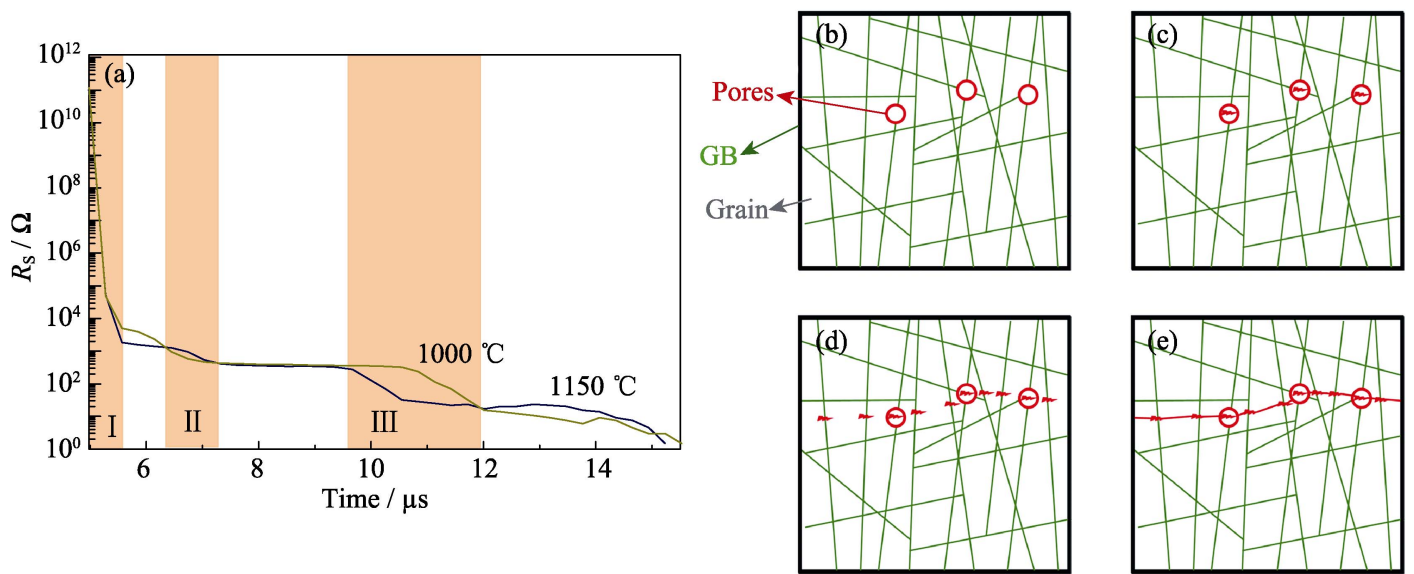

图 5 陶瓷电阻变化曲线(a)和击穿过程的图解示意图(b) (e)

Fig. 5 Resistance change during breakdown process (a) and schematic diagrams of breakdown processes (b)-(e)

图 5(b) (e)为 $\mathrm{ZnO}$ 压敏陶瓷在脉冲电场下的击 穿过程示意图，图中圆圈代表陶瓷体中的气孔，线 条代表晶界，由线条交织围成的块状区域代表晶 粒。图 5(c)为缺陷击穿阶段, 在脉冲电场的作用下, 能量首先集中于 $\mathrm{ZnO}$ 压敏陶瓷体内部的气孔，此时， 导电通道开始形成。图 5(d)为晶界击穿阶段, 气孔 击穿后, 施加在陶瓷晶界上的电场增大, 晶界附近 的载流子浓度逐渐上升, 其累加的热效应导致晶界 被击穿, 导电通道拓展至晶界。图 5(e) 为晶粒击穿 阶段, 在气孔和晶界均被击穿后, 能量全部集中于 晶粒，使晶粒发生击穿现象，最终陶瓷体被完全击 穿。综上所述, 可将陶瓷被击穿的先后顺序归纳为: 气孔击穿 $\rightarrow$ 晶界击穿 $\rightarrow$ 晶粒击穿。此结果与文献上 报道的 PZT95/5 陶瓷击穿过程 ${ }^{[6]}$ 有相似之处。多晶 陶瓷材料内部的缺陷在电场加载下, 会出现局域的 电场增强，从而首先击穿的是存在气孔的局部区域, 之后再是陶瓷本身的击穿。

从击穿时间来看，图 4 中三个击穿区的击穿时 间大小关系为：击穿区 7>击穿区 5>击穿区 3 。 $1000{ }^{\circ} \mathrm{C}$ 样品击穿的总时间以及对应的击穿区 5 和击 穿区 7 时间均长于 $1150{ }^{\circ} \mathrm{C}$ 样品, 但二者击穿区 3 时 间几乎相等。方波持续时间越长, 方波能量越大。
由此可以认为, 在 $\mathrm{ZnO}$ 压敏陶瓷中, 各部分能量耐 受能力大小关系为: 晶粒 $>$ 晶界 $>$ 气孔。对比两个试 样， $1000{ }^{\circ} \mathrm{C}$ 样品晶界、晶粒的能量耐受能力均大于 $1150{ }^{\circ} \mathrm{C}$ 样品, 而二者气孔的能量耐受能力相仿。一 般来说, 陶瓷体内部电阻分布的均匀性是影响能量 耐受能力的主要因素 ${ }^{[24-25]}$ 。随着烧结温度升高, 气 孔的体积增大, 但在配方比例和制备流程相同的情 况下，可以认为气孔分布的均匀性差异不大。而烧 结温度的升高同样会使部分晶粒出现二次结晶的现 象, 导致一些晶界的消失和极大晶粒的出现, 劣化 了晶界和晶粒电阻的均匀性, 使晶界和晶粒的能量 耐受能力下降, 击穿时间缩短。基于图 4(a) (b)所示 击穿电压波形变化图, 进一步研究了不同烧结温度 的 $\mathrm{ZnO}$ 压敏陶瓷在三个击穿过程中的速度差异，对 应图 4(a) (b)中的击穿区 $(3,5,7)$, 如图 6 所示。发现 $1150{ }^{\circ} \mathrm{C}$ 样品的气孔击穿速度大约为 $8.8 \mathrm{kV} /(\mathrm{mm} \cdot \mu \mathrm{s})$ 大于 $1000{ }^{\circ} \mathrm{C}$ 样品的 $2.1 \mathrm{kV} /(\mathrm{mm} \cdot \mu \mathrm{s})$, 而二者晶界和 晶粒的击穿速度没有明显差异。可以认为 $1150{ }^{\circ} \mathrm{C}$ 样 品中更大的气孔体积造成了气孔结构附近更大的电 势分布梯度，从而使局部电场更为集中，能量聚集 得更快，因此击穿速度更快。而晶界和晶粒的击 穿是由于电场继续增加后载流子所产生热效应的累
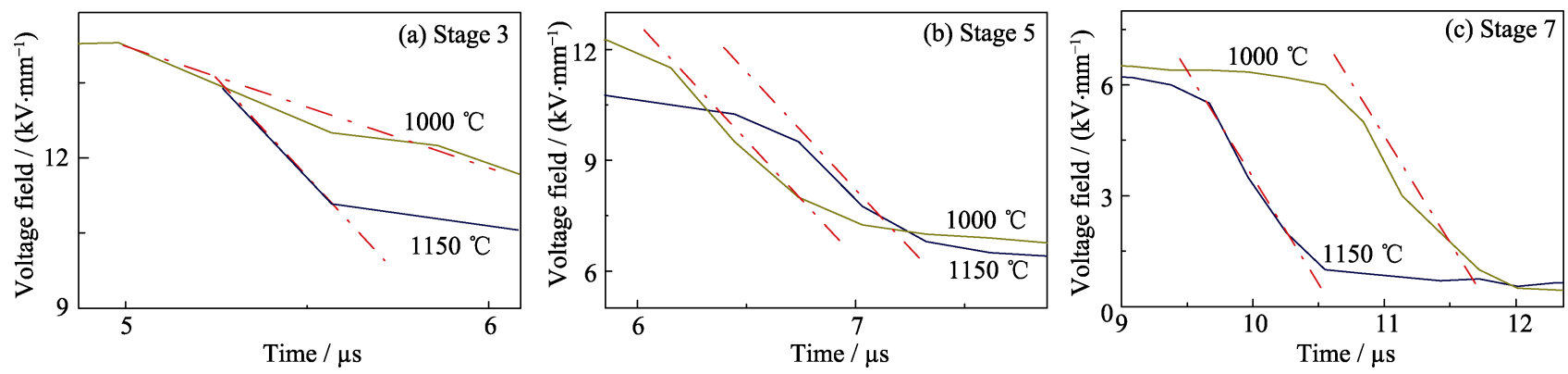

图 6 两个样品在气孔击穿(a)、晶界击穿(b)和晶粒击穿(c)时的对比图

Fig. 6 Voltage changes during pores breakdown (a), grains boundary breakdown (b), and grains breaksdown (c) 
积 ${ }^{21]}$, 在组分和制备流程相同的前提下, 不同温度 烧结的样品其晶界和晶粒中的载流子浓度大致相同, 电流传导速度接近, 所以 1000 和 $1150{ }^{\circ} \mathrm{C}$ 样品在晶界 击穿区和晶粒击穿区的击穿速度是几乎一致的。

\section{3 结论}

本文对不同烧结温度 $\left(1000\right.$ 和 $\left.1150{ }^{\circ} \mathrm{C}\right)$ 的 $\mathrm{ZnO}$ 压敏陶瓷在脉冲电场下的动态击穿过程进行了初 探。通过其压降大小的变化, 确定了其体击穿的时 间步骤为气孔击穿 $\rightarrow$ 晶界击穿 $\rightarrow$ 晶粒击穿, 并通过 对击穿曲线的进一步分析, 认定影响各击穿区域击 穿时间的因素为对应结构电阻分布的均匀性, 各部 分能量耐受能力大小关系为: 晶粒 $>$ 晶界 $>$ 气孔。不 同烧结温度下, 样品的气孔击穿时间保持一致, 而 晶界和晶粒的击穿时间随样品烧结温度的升高而降 低。影响各击穿区域击穿速度的因素为电流在相应 击穿通道的累积速度, 随着样品的烧结温度的升高, 气孔的击穿速度加快, 晶界及晶粒的击穿速度保持 不变。

\section{参考文献}

[1] MATSUOKA M. Nonohmic properties of zinc oxide ceramics. J. Appl. Phys., 1971, 10(6): 736-746.

[2] CHEN H C, ZANG G Z, WANG J F, et al. Effect of $\mathrm{MgSiO}_{3}$ on the electrical properties of $\mathrm{ZnO}$-based varistors. Eleectronic Components and Materials, 2006, 25(8): 19-21.

[3] HE J L, HU J, MENG B W, et al. Requirement of ultra-high voltage GIS arrester to voltage gradient of metal-oxide varistor. Sci. China, Ser., 2009, 52(2): 450-455.

[4] ZHANG F P, DU J M, LIU Y S, et al. Failure mechanism of PZT95/5 under direct current and plused electric field. Acta Phys. Sin., 2011, 60(5): 708-712.

[5] CHEN Y L, ZHANG F, GE G B, et al. Observation of breakdown phenomenon of middle-high voltage ceramic capacitor materials by meaans of high-speed video camera. Electronic Components and Materials, 2013, 32(3): 37-41.

[6] LIU Y, YANG J, LI X, et al. Resistance of $\mathrm{Pb}_{0.99}\left(\mathrm{Zr}_{0.95} \mathrm{Ti}_{0.05}\right)_{0.98} \mathrm{Nb}_{0.02} \mathrm{O}_{3}$ under high voltage microsecond pulse induced breakdown. Acta Phys. Sin., 2017, 66(11): 299-304.

[7] CHU S Y, YAN T M, CHEN S L. Analysis of $\mathrm{ZnO}$ varistors prepared by the Sol-Gel method. Ceram. Int., 2000, 26(7): 733-737.

[8] LIU T T, WANG M H, ZHANG H P, et al. Sol-Gel synthesis of doped nanocrystalline $\mathrm{ZnO}$ powders using xanthan gum and varistor properties study. J. Mater. Sci-Mater., 2015, 26(11): 9056-9062.

[9] JIANG S, WANG Y, ZHANG X, et al. Effect of Zn doping on stability of $\mathrm{ZnO}$ varistors under high pulse-current stress. Ceram. Int., 2015, 41(9): 11611-11617.

[10] ZHAO H, HU J, CHEN S, et al. Improving age stability and energy absorption capabilities of $\mathrm{ZnO}$ varistors ceramics. Ceram. Int., 2016, 42(15): 17880-17883.

[11] ZHAO H, HU J, CHEN S, et al. Tailoring the high-impulse current discharge capability of $\mathrm{ZnO}$ varistor ceramics by doping with $\mathrm{Ga}_{2} \mathrm{O}_{3}$. Ceram. Int., 2016, 42(4): 5582-5586.

[12] SHEN J, JIANG S, XU Y, et al. Boron and sodium co-doped ZnO varistor with high stability of pulse current surge. J. Alloy. Compd., 2017, 728: 368-375.

[13] MENG P, GU S, WANG J, et al. Improving electrical properties of multiple dopant $\mathrm{ZnO}$ varistor by doping with indium and gallium. Ceram. Int., 2017, 44(1): 1168-1171.

[14] LONG W, HU J, LIU J, et al. Effects of cobalt doping on the electrical characteristics of Al-doped $\mathrm{ZnO}$ varistors. Mater. Lett., 2010, 64(9): 1081-1084.

[15] XU Y W, HE Z W, XU Z, et al. Effects of $\mathrm{Co}_{2} \mathrm{O}_{3}$ and $\mathrm{MnCO}_{3}$ addition on the electrical properties of low-voltage $\mathrm{ZnO}$ varistor. Journal of Materials Science and Engineering, 2005, 23(4): 53-56.

[16] PAN X J, ZHANG Z X, WANG T, et al. Room temperature photoluminescence from nanocrystalline GaN:Er film prepared by sputtering. Acta Phys. Sin., 2008, 57(6): 3786-3790.

[17] MUKAE K, TSUDA K, NAMASAWA I. Non-Ohmic properties of $\mathrm{ZnO}$-rare earth metal oxide- $\mathrm{Co}_{3} \mathrm{O}_{4}$ ceramics. Japn. J. Appl. Phys., 2014, 16(8): 1361-1368.

[18] WU J Z, GUO Y Y. Influences of additives on properites of nonlinear ZnO ceramics. J. Inorg. Mater., 1991, 6(2): 185-192.

[19] KIM J, KIMURA T, YAMAGUCHI T. Microstructure development in $\mathrm{Sb}_{2} \mathrm{O}_{3}$-doped $\mathrm{ZnO}$. J. Mater. Sci., 1989, 24(7): 2581-2586.

[20] OTT J, LORENZ A, HARRER M, et al. The influence of $\mathrm{Bi}_{2} \mathrm{O}_{3}$ and $\mathrm{Sb}_{2} \mathrm{O}_{3}$ on the electrical properties of $\mathrm{ZnO}$-based varistors. $J$. Electroceram., 2001, 6(2): 135-146.

[21] JIANG Y X, WANG S Z, HE H L. Channel induced electromechanical breakdown model for porous PZT95/5 ceramic in quasi-static electric fields. Chin. J. High. Press. Phy., 2014, 28(6): 680-685.

[22] ZHANG B Y, YAO J C, CHANG A M, et al. Preparation and complex impedance analysis of $\mathrm{Mn}_{2.1} \mathrm{Ni}_{0.9} \mathrm{O}_{4}$ negative temperature coefficient ceramics. Electronic Components and Materials, 2013, 32(7): $1-5$.

[23] HAO S E, WEI Y D, KUANG C. Effects of $\mathrm{Gd}_{2} \mathrm{O}_{3}$ doping on electric characteristics of $\mathrm{BaTiO}_{3}$ ceramics. Fine Chemicals, 2002, 19(12): 717-719.

[24] BARTKOWIAK M, COMBER M G, MAHAN G D. Influence of nonuniformity of $\mathrm{ZnO}$ varistors on their energy absorption capability. IEEE T. Power Deliver, 2001, 16(4): 591-598.

[25] HE J L, HU J. Discussions on nonuniformity of energy absorption capabilities of $\mathrm{ZnO}$ varistors. IEEE T. Power Deliver, 2007, 22(3): 1523-1532. 